\title{
Lens Capsule HSPG-Perlecan Regulates Lens Fibre Differentiation during Chick Embryo Development
}

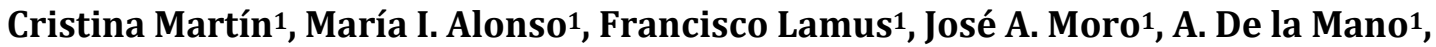 \\ José M. Fernandez ${ }^{2}$, Alberto Caballero ${ }^{1}$, Ángel Gato ${ }^{{ }^{*}}$ \\ ${ }^{1}$ Departamento de Anatomía y Radiología, Laboratorio de Desarrollo y Teratología del Sistema Nervioso. Instituto de \\ Neurociencias de Castilla y León (INCYL); Facultad de Medicina, Universidad de Valladolid, Valladolid, España \\ ${ }^{2}$ Departamento de Biología Celular, Histología y Farmacología; Facultad de Medicina, Universidad de Valladolid, Valladolid, \\ Spain \\ Email: *gato@med.uva.es
}

How to cite this paper: Martín, C., Alonso, M.I., Lamus, F., Moro, J.A., De la Mano, A., Fernandez, J.M., Caballero, A. and Gato, Á. (2017) Lens Capsule HSPG-Perlecan Regulates Lens Fibre Differentiation during Chick Embryo Development. Open Journal of Veterinary Medicine, 7, 9-22. https://doi.org/10.4236/ojvm.2017.72002

Received: October 18, 2016 Accepted: February 25, 2017 Published: February 28, 2017

Copyright $\odot 2017$ by authors and Scientific Research Publishing Inc. This work is licensed under the Creative Commons Attribution International License (CC BY 4.0).

http://creativecommons.org/licenses/by/4.0/

\begin{abstract}
Lens fibre differentiation is a life-long process related with lens transparency, and is particularly intense during development, being related with an FGF-2 antero-posterior gradient at the equator level as the main growth factor involved which has been related with the basal membrane of the lens anlagen known as "Lens capsule". However the lens fibre differentiation induced by FGF2 depends, as in other biological systems, on the local bioavailability of FGF-2 regulated by their relationship with extracellular matrix molecules as Heparan Sulphate Proteoglycans. Here, we try to clarify how Perlecan (a heparan sulphate proteoglycan specific from basement membranes) is involved in lens fibre differentiation at earliest stages of eye development. Our results show that Perlecan, is a major component in the lens capsule during the earliest stages of lens development in chick embryos being present during lens plate induction, lens vesicle stage and the onset of lens fibre differentiation. In order to demonstrate a direct involvement of HSPG-Perlecan in lens fibre differentiation, we generate depleted lenses by HSPG-Perlecan synthesis disruption and specific enzymatic digestion. The HSPG-Perlecan depleted lens show a significant delay or abolition in the lens fibre differentiation which remains in an immature cells displaying DNA synthesis in the posterior epithelium and a decrease in FGF2 lens expression. These data support the hypothesis that lens capsule HSPG-Perlecan is a key molecule involved in lens fibre differentiation during development, probably by involvement in FGF-2 biodisponibility.
\end{abstract}

\section{Keywords}

Eye Development, Lens Fibre Differentiation, HSPG-Perlecan, FGF-2, Chick Embryo 


\section{Introduction}

From early development, lens vesicle epithelial cells undergo a differentiation process towards primary lens fibre, and these cellular dynamics remain throughout life with cell replication in the anterior epithelium and migration through the equatorial (peripheral) area towards the posterior epithelium, in which cells differentiate in lens fibres [1]. This cellular behaviour associated with lens fibre differentiation is restricted later at foetal and adult periods, to the equatorial area. Despite the involvement of growth factors like IGF-I and TGF- $\beta$ has been reported in lens fibre differentiation [2] [3] [4], the FGF-2 remains as the strongest candidate in the regulation of lens fibre differentiation during development both in rodents and chick [5] [6].

Currently, however, modulators of growth factor signalling in lens fibre differentiation induced by FGF-2 are seen to be influential and two kinds of mechanisms have been proposed. One is based on molecules able to repress the FGF2 activity such as "sprouty" and "sef" families of genes, which are strongly expressed in the lens anterior epithelium exerting a repressive action in FGF-2 lens cells signalling and maintain their epithelial state [7] [8]. The other mechanism proposed is that of regulation of FGF-2 bioavailability and receptor activation by the extracellular matrix proteoglycans, as described for Heparan Sulphate proteoglycans in many biological systems [9] [10]. In this context, there are previous reports of the presence and involvement of proteoglycans in several aspects of early lens development [11] [12]; in addition, special attention has been given to the influence of the CSPG and HSPG of the lens capsule in lens fibre differentiation in birds and mammals [13] [14], suggesting that HSPG from the lens capsule might be involved in lens fibre differentiation by FGF-2 activity regulation [15]. However, experimental support for this relation is necessary.

In this paper, we show the expression pattern of Perlecan A besal membrane HSPG in the lens capsule at the earliest stages of chick embryo lens development, and we provide experimental support of direct relation between lens capsule HSPG-Perlecan and lens fibre differentiation during chick eye development.

\section{Materials and Methods}

\subsection{Obtaining Chick Embryos and Experimental Approach}

Fertile white hybrid hens eggs were incubated at $38^{\circ} \mathrm{C}$ in a humidified atmosphere to obtain chick embryos at different developmental stages, ranging from 13 to 25 H.H. [16], in order to study the expression pattern of HSPG-Perlecan. A total of 20 embryos were incubated up to stage 17 H.H. (corresponding to that of the lens vesicle, prior to differentiation of lens fibres), at which a single dose of $24 \mu \mathrm{l}$ $\beta$-D-xyloside (a sulphated proteoglycans synthesis disruptor) $4 \mathrm{mM}$ (Sigma) was injected subgerminally with a Hamilton microsyringe [12], and the eggs were reincubated for 24 hours until stage 23 H.H. (corresponding to the development of lens fibres). Controls (15 embryos) used in the experiment were treated with a sterile saline solution with the same procedure. All the animal handling and procedures were supervised by the research and welfare service from the Valla- 
dolid University.

A total of 30 embryos were treated with heparinase as follows: once embryos reached stage 17 H.H., a small opening was made in the shell to expose the right eye of the embryo, after which the vitelline membrane was cut with tungsten needle and $12 \mathrm{nl}$ of a heparinase II solution from Flavobacterium heparinum (Sigma) (10 U of enzyme dissolved in $25 \mu \mathrm{l}$ of PBS), was microinjected into the subectodermal space in the area of the lens equator. As controls, we microinjected 15 embryos with the same amount of heat-inactivated enzyme as for the enzyme treated. Microinjection was performed with a microinjector (Medical Systems Corp, Greenvale, NY 11548, U.S.A, PLI-100). Following this, the eggs were sealed and re-incubated for a period of 18 hours until stage $21 \mathrm{H} . \mathrm{H}$.

In both cases ( $\beta$-D-xyloside and heparinase II treatment) a single dose of 200 $\mathrm{nl}$ of $\mathrm{BrdU}(0.3 \%$ solution) was microinjected in the outflow of the heart just 1 hour before the end of the reincubation period. The embryos were then removed from the extraembryonic membranes and fixed for $1 \mathrm{hr}$ in Carnoy's.

\subsection{Immunohistochemistry}

Histological sections $(8 \mu)$ from five different lens anlagen at each stage were washed in phosphate-buffered saline (PBS), pre-incubated with normal horse serum (1/20 in PBS) and incubated overnight at room temperature with a 1:1000 dilution of Anti Heparan Sulphate-Perlecan monoclonal antibody (Upstate Biotechnology). After two washings in PBS, the sections were reincubated for 30 minutes in fluorescein-conjugated goat anti-rat Ig-G FITC conjugate (Sigma) as a secondary antibody, mounted in Aquamount (Gurr) and examined under a Leica SPE laser confocal microscope. Control sections were prepared as described above but pre-immune serum was used as the primary antibody and no labelling was observed.

In others cases, lens anlagen histological sections $(8 \mu \mathrm{m})$ were deparaffinised and BrdU was detected following standard procedures [17]. The sections were incubated in a solution containing a $1 / 100$ dilution of monoclonal antibody to BrdU (Dako) for 30 minutes at room temperature. An avidin-extravidin system conjugated to peroxidase (mouse anti-rabbit $1 / 20$ for 30 minutes and extravidin 1/20 for 10 minutes; Sigma) was used to detect the primary antibody and was developed with DAB. A Nikon microphot-FXA photomicroscope was employed to visualize and photograph the preparations.

In other cases, lens anlagen frozen sections $(5 \mu \mathrm{m})$ were used to detect FGF2 following standard procedures. The sections were incubated in a solution containing a 1/1000 dilution of monoclonal antibody to FGF2 (anti-bovine FGF2 antibody from Sigma) for 30 minutes at room temperature. An ExtrAvidin stainig Kit antimouse system conjugated to peroxidase (from Sigma) was used to detect the primary antibody and was developed with DAB. A Nikon microphot-FXA photomicroscope was employed to visualize and photograph the preparations.

Finally, lens anlagen histological sections $(8 \mu \mathrm{m})$ were deparaffinised, dehydrated and stained with Haematoxilin-Eosin technique following standard pro- 
cedures. We used a Nikon microphot-FXA photomicroscope to visualize and photograph the preparations.

\section{Results}

\subsection{Immunohistochemical Detection of HSPG-Perlecan during Early Lens Development}

In this study we have used a monoclonal antibody which recognizes Perlecan, a type of Heparan Sulphate Proteoglycan present in basement membranes [18] [19] [20]. As we show in Figure 1, the antibody specifically labels basement membranes in the eye anlagen, including the surface ectoderm, lens and optic cup, at different stages of development.

At stage14 H.H., we show (Figure 1(a)) intense immunolabelling in the basement membrane of both the lens placode (similar to surface ectoderm basement membrane labelling) and the optic cup, although in some case both basement membranes seem to be in direct contact. During lens placode invagination (stages 15 - 16 H.H.) (Figure 1(b) and Figure 1(c)) there is still intense immunolabelling on the basement surface of the lens epithelium, however during this period intensity on the basement surface of the optic cup decreases. At these stages it is possible to observe several areas in which both basement membranes (lens and optic cup) are in close contact. A similar disposition in terms of Perlecan immunomarking can be seen at stages $16-17$ H.H. (Figure 1(d)); furthermore, at these stages it can be observed how basement membrane immunolabelling in the lens anterior epithelium is restored as the final step in lens isolation from the ectodermal surface.

From stages 18 to $25 \mathrm{H} . \mathrm{H}$. (Figure $1(\mathrm{e})-(\mathrm{h})$ ), the lens capsule is the structure with the most intense immunolabelling in the eye anlage, appearing as a strong, continuous and apparently uniform layer on the basement side of the lens. At the beginning of lens fibre differentiation we cannot appreciate any labelling in the anterior and posterior epithelium cells.

As can be observed in Figure 1(e) and Figure 1(f), at these stages of eye development (E18 - 20 H.H.) immunolabelling on the surface ectoderm and neural retina decrease considerably; however, in some sections a point of contact can be perceived between the neural retina at the margin of the optic cup and the basement membrane of the lens equator (Figure $1(\mathrm{~h})$ ), both labelled by the Perlecan antibody. Finally, we detect the "pecten" (Figure 1(g)) as another structure showing strong immunolabelling during chick eye development.

\subsection{Disruption of Lens HSPG Alters Normal Lens Fiber Growth and Differentiation}

With the aim to test the role of Heparan Sulphate-Perlecan proteoglycan on lens development, especially in the differentiation of lens fibres from epithelial cells, we carried out two experimental approaches: first, we used $\beta$-Dxyloside, a xylosiltransferase substrate which disrupts the assembly of sulphated proteoglycans including different forms of HSPG [21]; secondly, we used Heparinase 

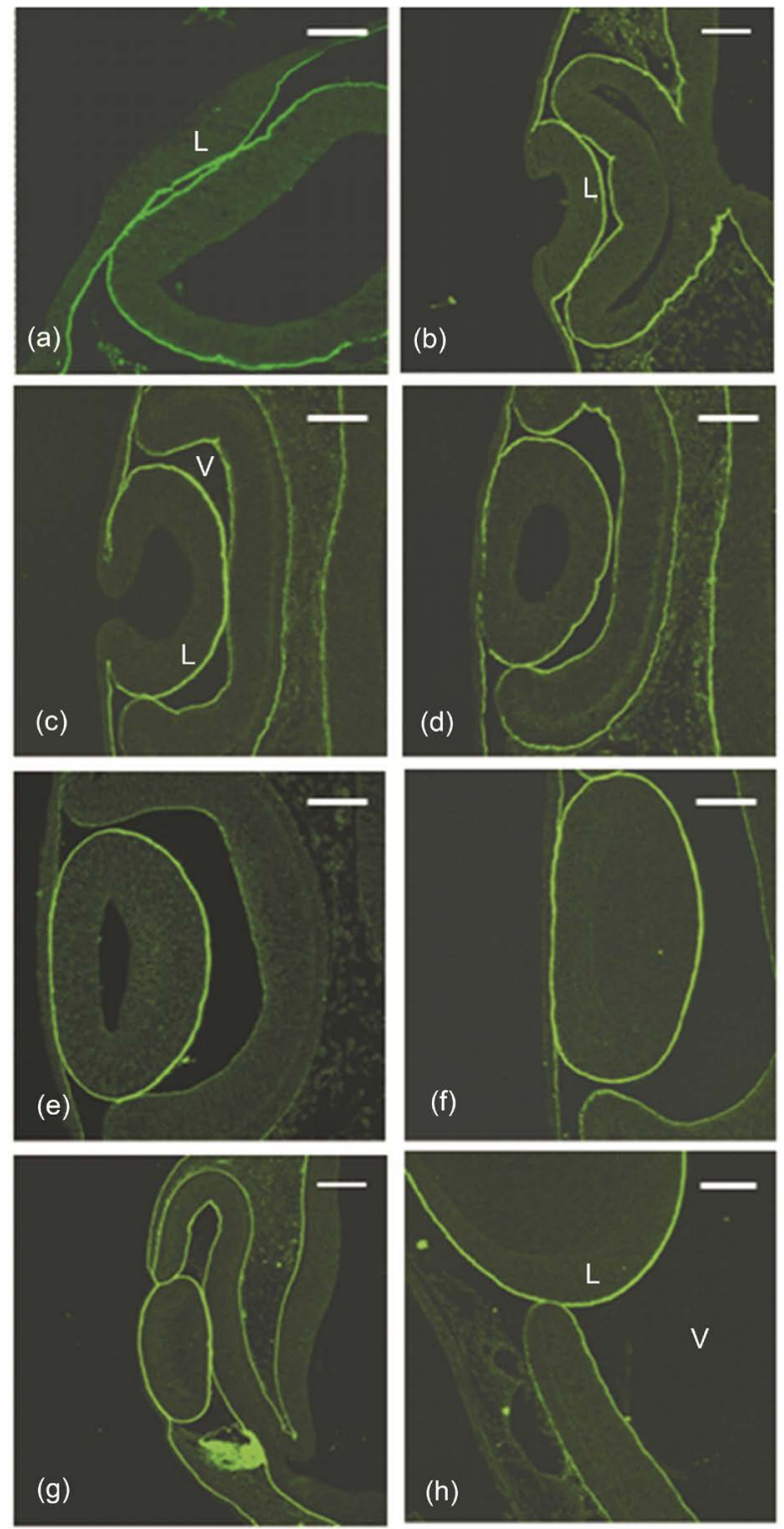

Figure 1. Immunohistochemistry micrographs of chick embryo lens primordium sections, processed with anti-Heparan-Sulphate-Perlecan antibody and a conjugate secondary antibody with fluorescein isothiocyanate (FITC). The images show the HSPG-Perlecan expression patterns during lens development. (a) (E14 H.H. lens placode). (b) (c) (E15-16 H.H. lens placode invagination). (d) (E16-17 H.H. lens vesicle). (e) (E18H.H.), (f) (E20 H.H.) and (g) (h) E25 H.H.) showing the lens fibre differentiation process. (a), (c), (d), (e), (f): Scale Bar $=50 \mu \mathrm{m}$; (b), (g): Scale Bar $=100 \mu \mathrm{m} ; \mathrm{H}$ : Scale Bar $=30 \mu \mathrm{m}$. L: Lens. V: Vitreous.

type II, which specifically digest Heparan Sulphate including Perlecan [22]. Both treatments were administered at stage 17 H.H., which corresponds with the lens vesicle stage, before initial lens fibre differentiation, and were maintained until the $23 \mathrm{H} . \mathrm{H}$., when the histological structure of the lens anlagen (Figure 2(a)) shows a polarized lens with a thin anterior epithelium, a transitional equatorial (marginal) area and a thick posterior epithelium displaying the elongated primary fibres with a central nucleus, closing the inner cavity. 
As seen in Figure 2(d), BrdU immunolabelling showed the nucleus with DNA synthesis activity (which at these stages of development could be assimilated to mitotic activity) in the anterior epithelium, in which the homogeneous presence of a layer of BrdU-positive nucleus reaching the equatorial area could be observed; however, in the posterior epithelium, there was a total absence of BrdU-positive nucleus. This mitotic pattern was consistent with the dynamic cellular behaviour described during lens development [23] with cellular replication in the anterior epithelium, migration through the equatorial zone and differentiation in the posterior epithelium. As we described before, the eyes of stage $23 \mathrm{H}$.H. controls showed intense immunolabelling with the anti-perlecan antibody in the lens capsule (Figure 1(f)). Finally, lens capsule immunodetection of FGF2 show the absence of labelling in the anterior epithelium and a ubiquitous label in the lens fibre which was more intense in the posterior part of the lens capsule (Figure $2(j))$.

All the $\beta$-Dxyloside treated embryos showed a severe, specific disruption in lens anlagen development. The histological study shows the lens anlagen with an undifferentiated posterior epithelium with no lens fibre growth, resembling the lens vesicle stage (Figure $2(\mathrm{~b})$ ). In other cases (Figure $2(\mathrm{c})$ ), some areas of the posterior epithelium show a partial lens fibre growth while others remain undifferentiated and the lens vesicle cavity remains uncollapsed. In the $\beta$-Dxyloside treated embryos, BrdU immunodetection (Figure 1(e)) shows a clear disruption in the mitotic behaviour of the lens anlagen cells, with an increased number of positive nuclei in the anterior epithelium which appear disposed in several layers and a significant number of positive nuclei in the posterior epithelium which seems to remain in an undifferentiated state. In order to test the efficiency of $\beta$-Dxyloside treatment in HSPG-Perlecan lens capsule disruption, we studied the expression of this proteoglycan by immunohistohemistry. Figure $2(\mathrm{~g})$ shows how, in the $\beta$-Dxyloside treated lens, there was a large decrease in Perlecan immunolabelling compared with the controls (Figure 2(f)), displaying a non-homogeneous distribution and extensive zones with no Perlecan expression, mainly located in the posterior epithelium. Finally the FGF2 lens anlagen immunolabelling was dramatically reduced in the fibre cells and posterior part of the lens capsule in $\beta$-Dxyloside treated lens (Figure $2(\mathrm{k})$ ) with respect to that of the control embryos (Figure $2(\mathrm{~h})$ ).

In order to confirm the specificity of our results, we made a selective enzymatic digestion of HSPG (Perlecan) in the developing lens with Heparinase II microinjection in the lens anlagen equator. Heparinase II treated embryos (Figure 3(b)) show a hystolological appearance of the lens anlagen with the same alterations as in the $\beta$-D-Xyloside treated embryos, namely, a reduced thickness in the posterior epithelium, with a dramatic decrease in the length of the lens fibre cytoplasm (with the lens vesicle cavity remaining completely uncollapsed) compared with the control (Figure 3(a)); occasionally the Heparinase II treated embryos presented a completely undifferentiated posterior epithelium with no signs of lens fibre cytoplasmic growth (Figure $3(\mathrm{c})$ ). Compared with the control 
(a)

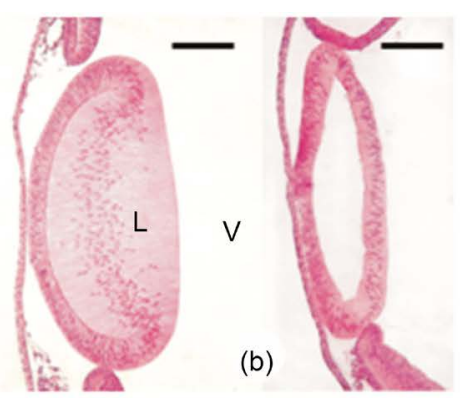

(c)

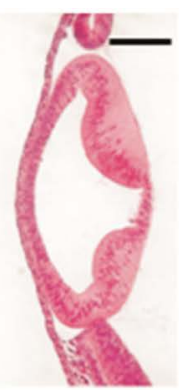

(d)

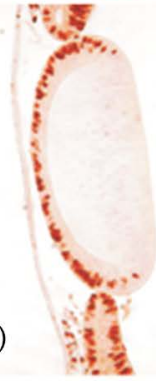

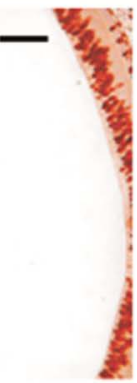

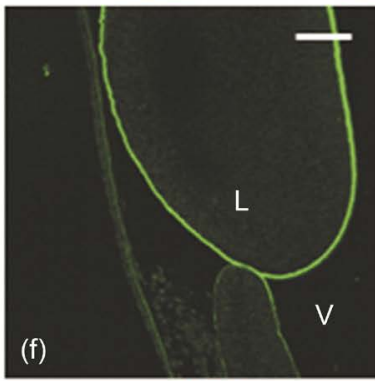

(e)
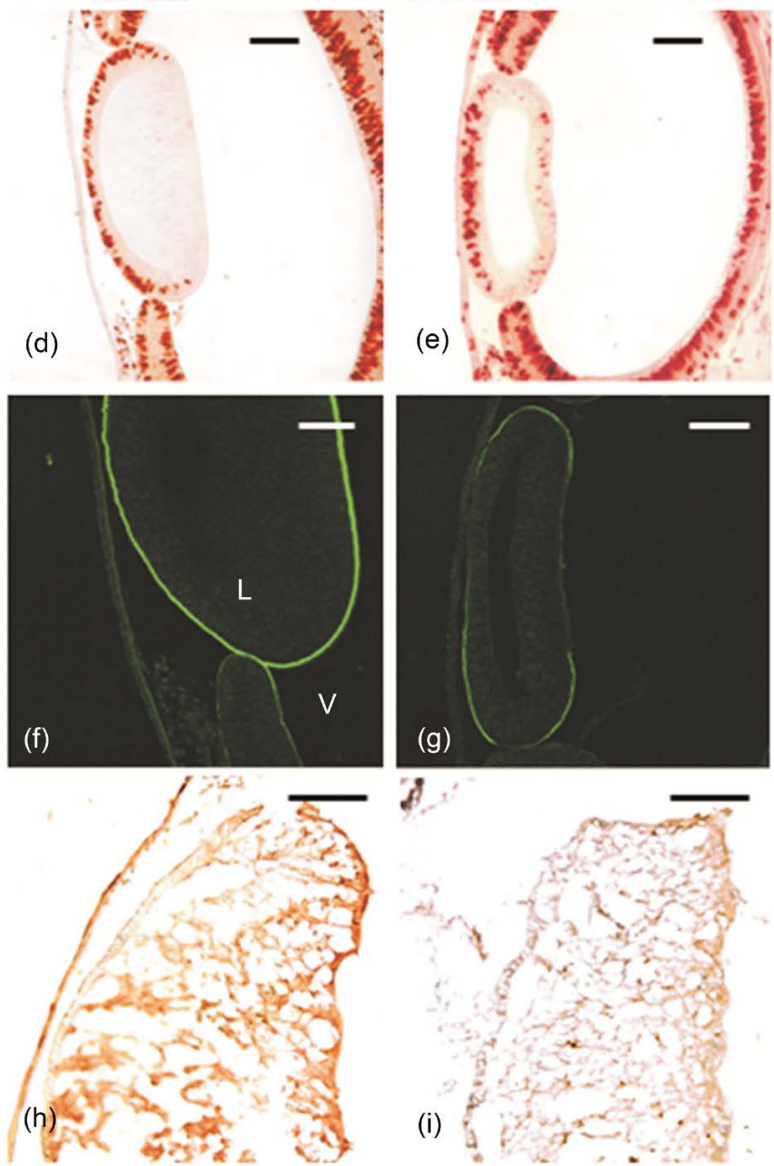

Figure 2. $\beta$-D-Xyloside treated lens anlagen. Micrographs of middle sections of chick lens anlagen of E23 H.H. embryos. Haematoxylin-Eosin stained sections of a control (a) and a $\beta$-D-Xyloside-treated embryo (b) (c) showing disruption in lens fibre differentiation. Cell replication detected by BrdU immunolabelling in a control (d) and a $\beta$-D-Xyloside-treated embryo (e); note the presence of labelled nucleus in the posterior epithelium. Anti-Heparansulphate-Perlecan antibody fluorescein-labelled immunohistochemistry of a control (f) and a $\beta$-D-Xyloside-treated embryo (g) showing a clear decrease in HSPG- Perlecan expression in the lens capsule. Anti FGF2 lens capsule immunolabelling of a control $(\mathrm{h})$ and a $\beta$-D-Xyloside-treated embryo (i) showing a dramatic lost of (a, b, c, d, e) and g: Scale Bar $=50 \mu \mathrm{m}$. f: Scale Bar $=30 \mu \mathrm{m}$. h and i: Scale Bar $=100 \mu \mathrm{m}$. L: Lens. V: vitreous.

embryos (Figure 3(d)), BrdU immunodetection in the Heparinase II treated lens showed (Figure 3(e)) a greater number of BrdU positive nucleus in the anterior epithelium arranged in several layers and the abnormal presence of BrdU positive nucleus in the undifferentiated posterior epithelium. In order to test the ef- 
fectiveness of Heparinase II enzymatic activity on HSPG-Perlecan in the chick embryo lens anlagen, we made an immunohistochemical study, which shows that the enzyme treated lens (Figure 3(g)) had greatly reduced HSPG-Perlecan in the lens capsule compared with the control embryos (Figure 3(f)), showing a non homogeneous labelling pattern with several areas of no expression of Perlecan, almost in the posterior capsule. Finally, as is showed in Figure 3(h), a dramatic decrease in FGF2 expression was detected, both in the lens fibres and in the posterior lens capsule, in the Heparinase II treated lens in comparison with the control embryos (Figure 3(i)).

\section{Discussion}

Our results demonstrate that Perlecan, a macromolecule specific for basement membranes, is a ubiquitous and permanent component of the lens capsule during the first stages of lens fibre differentiation. Our results also strongly suggest that Perlecan is involved in regulating lens cell behaviour, playing a key role in lens fibre differentiation, probably in association with lens capsule FGF-2 activity.

The lens capsule has been described as a specific extracellular matrix structure which isolates the lens from its surroundings, and has been shown to be a key structure in controlling lens cell behaviour, regulating the outside-inside traffic signals (Danysh [15]. Here we described the presence of Perlecan in chick embryo lens at earliest stages of development which include the beginning of the lens fibre differentiation. Although other proteoglycans have been shown to be present in the lens capsule, the main sulphated proteoglycan related with early lens development is HSPG [24], which has been proposed to be related with the control of lens cell behaviour given its growth factor binding properties [25] [14]. Previous studies by [26] have reported the presence of Perlecan in chick developing lens from morula to $17 \mathrm{H} . \mathrm{H}$. stage, so during lens plate induction and invagination to form the lens vesicle but this period does not include the beginning of the lens fibre differentiation; at these stages, Perlecan could be related with invagination morphogenetic mechanisms such as has been described in otic placode invagination [27]. Furthermore they describe a strong Perlecan expression in the lens without specific localization. Here we report a precise description of HSPG-Perlecan during lens vesicle period and also during lens fibre differentiation stages showing that Perlecan expression is restricted to the lens capsule.

The use of $\beta$-D xyloside or Heparinase II (specific for HSPG molecules) treatment in our experimental approach, with similar results, demonstrates that lens fibre differentiation is disrupted by the depletion of some kinds of lens HSPG. As we comment before, $\beta$-D xyloside disrupts the synthesis of sulphated proteoglycans [28], including Heparan sulphate and Chondroitin Sulphate Proteoglycans which also have shown to play several important roles during early eye development [13] [29] [30] [31] [32] [33], consequently, our results with $\beta$-D_xyloside treatment could be due, at least in part, to the disruption of other proteoglycans than HSPG. However, here we report experimental evidences that 


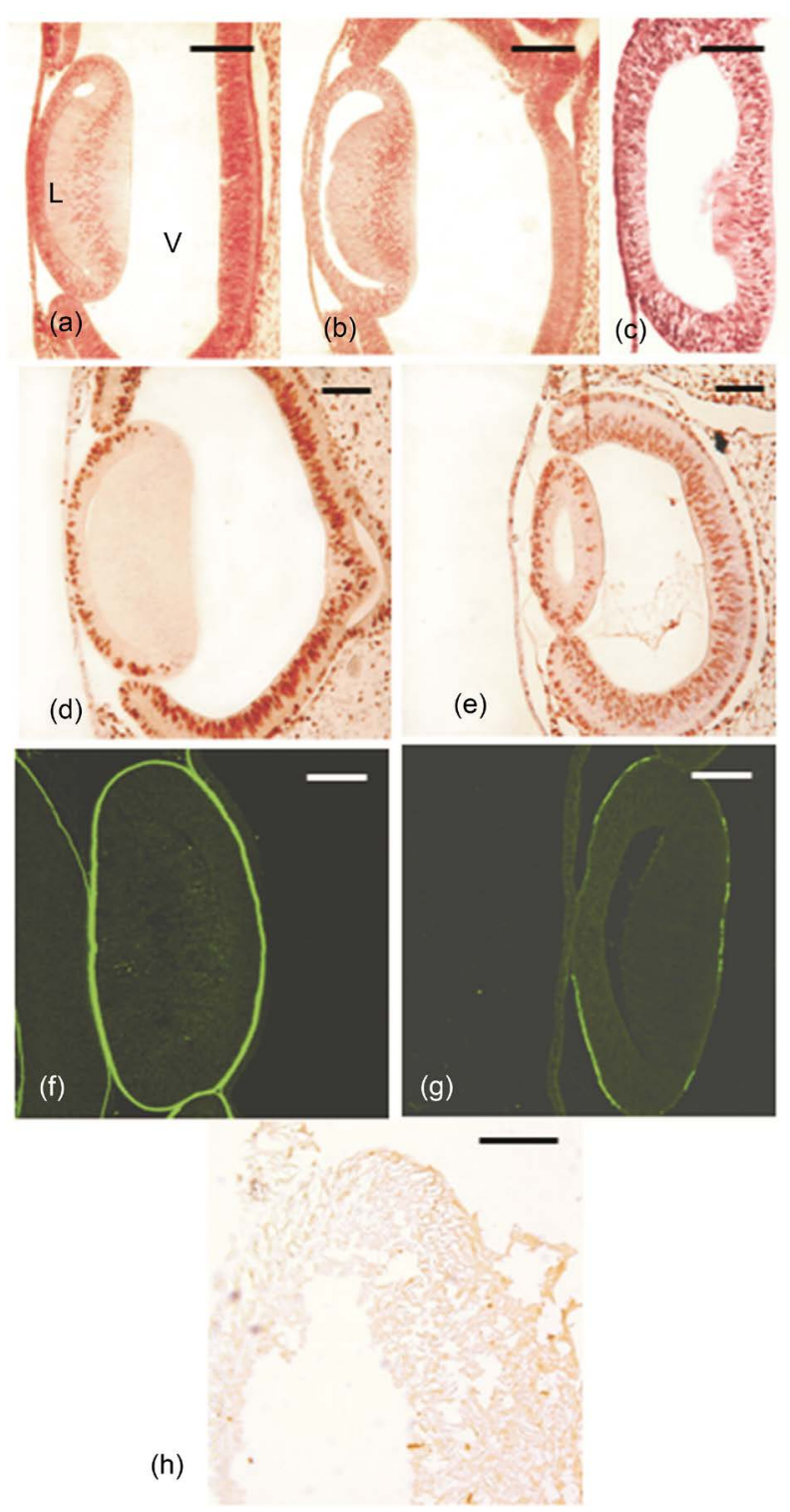

Figure 3. Heparinase II-treated lens anlagen: Micrographs of middle sections of chick lens anlagen of E23H.H. embryos. Haematoxylin-Eosin stained sections of a control (a) and a Heparinase II-treated embryo (b, c) showing disruption in lens fibre differentiation. Cell replication detected by BrdU immunolabelling in a control (d) and a Heparinase II-treated embryo (e); note the presence of labelled nucleus in the posterior epithelium. Anti-Heparan-Sulphate-Perlecan antibody fluorescein labelled immune- histochemistry of a control (f) and a Heparinase II-treated embryo (g) showing a clear decrease in HSPG-Perlecan expression in the lens capsule. Anti FGF2 lens capsule immunolabelling of a control (h) and a Heparinase II-treated embryo (h) showing a dramatic lost of FGF2. a, b and h:Scale Bar $=100 \mu \mathrm{m}$. c, d, e, f, g: Scale Bar $=50 \mu \mathrm{m}$. L: Lens. V: Vitreous.

the disruption of $\beta$-D_xyloside treatment made at 17 H.H. stage induces similar lens development disruption than the treatment made with Heparinase II and is coincident with Perlecan depletion in the lens capsule, so we can conclude that our results are generated by HSPG-Perlecan lens depletion.

Some studies described the presence of non specific HSPG located in the lens capsule during development [14] [15]. This work highlights the key role of Per- 
lecan (a complex macromolecule rich in heparan sulphate [34]), in lens fibre differentiation at early chick development. Supporting our results, an important role for Perlecan has been proposed by Rossi et al. [35] during late development in view of lens capsule degeneration in knock-out mice lacking the protein core of Perlecan. Perlecan has been also described as a key constituent of the lens capsule in adult cows [36] [37].

Our results also show that the disruption of Perlecan in the lens capsule of chick embryos, affects the whole range of cellular dynamics in the developing lens, showing an increase in the number of BrdU positive cells in the anterior epithelium, the presence of undifferentiated cells (BrdU positive) in the posterior epithelium and a deficit in lens fibre differentiation from the morphological (cytoplasmic elongation) point of view. Although it has been suggested that the different components of lens cell dynamics (replication, migration and differentiation) may depend on different and specific factors [38], our results suggest that all of them depend, at least initially, on common regulatory factors linked to the HSPG-Perlecan in lens capsule.

Given this important role of lens capsule Perlecan during development, an interesting question is the source of this macromolecule detected in the lens capsule. In this regard, Dong et al. [39] have demonstrated (by Perlecan m-RNA expression) that lens Perlecan, at the earliest stages of lens development, originates from the primary fibres in the posterior epithelium, and later Perlecan mRNA expression becomes restricted to lens fibres in the equatorial area, where all the cellular processes (replication, migration and differentiation) of lens differentiation take place. Our results show that the lens capsule is the principal structure containing Perlecan during early lens development, which strongly suggests that the primary lens fibres could play a key role in controlling new lens fibre differentiation, regulating the synthesis and "deposition" of Perlecan in the lens capsule.

Given the binding biological properties of HSPG containing molecules, as Perlecan, they are been proposed as molecules able to create a reservoir of growth factors in the surroundings of lens cells and in particular near to the region of lens fibre differentiation [25]. Our results show that Perlecan depleted lens, lost their FGF2 expression both in the lens capsule and in the fibre cells allowing us suppose that the relation of lens capsule Perlecan with lens fibre differentiation are also linked to the regulation of FGF2 concentration during lens developmet.

The lens is a biological structure in which two differentiated but interdependent cellular sub-populations coexist with transitional cellular behaviour (replication, migration and differentiation); the location is in the equatorial area and duration is life-long. A complex control involves many different molecular events in which FGF2 activity remains, to date, the main and initial regulatory mechanisms involved [36] [37] [40] [41] [43]. The traditional concept of FGF2 activity has been based on the gradient theory, which implies an increase in FGF2 concentration from anterior (surface) to posterior (deep) cellular populations, espe- 
cially in the equatorial area [42], Chamberlain [5] [43] [44]. However, in the last decade a new concept has developed in which the influence exerted by FGF2 on lens cellular behaviour is based on local gradients of FGF2 "activity". Recently, have been described new systems of complex regulation have in which the primary activity of and FGF-2 is activated and/or inhibited by secondary control mechanisms [45] [46]. These new theories propose that the undifferentiated stage of lens anterior epithelial cells is due to the expression of inhibitory genes such as Sprouty and Sef, which block the activity of the FGF-2 receptor [8] [39] [41], making a "low FGF2 activity" area.

Besides, several studies support the hypothesis of the reservoir of extracellular matrix growth factors, which in the lens capsule could also have an important regulatory influence on lens cell behaviour [36] [47]. Our results show that a depletion in lens capsule Perlecan during early lens chick development induces a near complete loss of FGF2 in the lens (both citoplasmic and capsular) and leads to severe disruption in lens dynamic and fibre differentiation. Supporting these results, Perlecan-linked HSPG in the adult cow lens capsule has been shown to regulate FGF2 activity, both increasing the local concentration of this growth factor near the lens fibres as well as enhancing FGF2 activity, probably by an FGF2 transdimerization which increases the efficiency of transmembrane signalling [34] [36] [48]. Moreover, the biological activity of lens capsule Perlecan could be regulated by an equilibrium between the synthesis of Perlecan by the lens fibres [39], which regulate the concentration of FGF2, and the activity of Metalloproteinases such as MMP2 in the lens capsule, which regulates the release of free FGF2 from this reservoir; this has been demonstrated in the adult cow lens [15] [36].

Taken together, all these data strongly support the hypothesis that the extracellular matrix organised as lens capsule plays a key role in lens fibre differentiation during development. This influence seems to be due in a great extent to their content in Perlecan which probably acts as FGF2 concentration regulator.

\section{Acknowledgements}

The authors thank to Dr. David Rixham for language translation assistance. This work was supported by the following Grants: Ministerio de Educación y Ciencia (BFU207/6516), Instituto de Salud Carlos III (PIO20961), Junta de Castilla y León (VA21A07, VA049/04, SAN673/VA15/08) and Federación Regional de Cajas de Ahorro.

\section{References}

[1] Chow, R.L. and Lang, R.A. (2001) Early Eye Development in Vertebrates. Annual Review of Cell and Developmental Biology, 17, 255-296. https://doi.org/10.1146/annurev.cellbio.17.1.255

[2] Bassas, L., Zelenka, P.S., Serrano, J. and de Pablo, F. (1987) Insulin and IGF Receptors Are Developmentally Regulated in the Chick Embryo Eye Lens. Experimental Cell Research, 168, 561-566.

https://doi.org/10.1016/0014-4827(87)90029-2 
[3] Bassnett, S. and Beebe, D.C. (1990) Localization of Insulin-Like Growth Factor-1 Binding Sites in the Embryonic Chicken Eye. Investigative Ophthalmology \& Visual Science, 31, 1637-1643.

[4] de Iongh, R.U., Lovicu, F.J., Overbeek, P.A., Schneider, M.D., Joya, J., Hardeman, E.D. and McAvoy, J.W. (2001) Requirement for TGFbeta Receptor Signaling during Terminal Lens Fiber Differentiation. Development, 128, 3995-4010.

[5] Chamberlain, C.G. and McAvoy, J.W. (1989) Induction of Lens Fibre Differentiation by Acidic and Basic Fibroblast Growth Factor (FGF). Growth Factors, 1, 125-134. https://doi.org/10.3109/08977198909029122

[6] Wang, Q.A., McAvoy, J.W. and Lovicu, F.J. (2010) Growth Factor Signaling in Vitreous Humor-Induced Lens Fiber Differentiation. Investigative Ophthalmology \& Visual Science, 51, 3599-3610. https://doi.org/10.1167/iovs.09-4797

[7] Kim, H.J. and Bar-Sagi, D. (2004) Modulation of Signalling by Sprouty: A Developing Story. Nature Reviews Molecular Cell Biology, 5, 441-450. https://doi.org/10.1038/nrm1400

[8] Boros, J., Newitt, P., Wang, Q., McAvoy, J.W. and Lovicu, F.J. (2006) Sef and Sprouty Expression in the Developing Ocular Lens: Implications for Regulating Lens Cell Proliferation and Differentiation. Seminars in Cell \& Developmental Biology, 17, 741-752. https://doi.org/10.1016/j.semcdb.2006.10.007

[9] Ruoslahti, E. and Yamaguchi, Y. (1991) Proteoglycans as Modulators of Growth Factor Activities. Cell, 64, 867-869. https://doi.org/10.1016/0092-8674(91)90308-L

[10] Goetz, R. and Mohammadi, M. (2013) Exploring Mechanisms of FGF Signalling through the Lens of Structural Biology. Nature Reviews Molecular Cell Biology, 14, 166-180. https://doi.org/10.1038/nrm3528

[11] Hendrix, R.W. and Zwaan, J. (1974) Changes in the Glycoprotein Concentration of the Extracellular Matrix between Lens and Optic Vesicle Associated with Early Lens Differentiation. Differentiation, 2, 357-362. https://doi.org/10.1111/j.1432-0436.1974.tb00371.x

[12] Gato, A., Martin, C., Alonso, M.I., Martinez-Alvarez, C. and Moro, J.A. (2001) Chondroitin Sulphate Proteoglycan Is Involved in Lens Vesicle Morphogenesis in Chick Embryos. Experimental Eye Research, 73, 469-478.

https://doi.org/10.1006/exer.2001.1060

[13] Bard, J.B.L. and Abbott, A.S. (1979) Matrices Containing Glycosaminoglycans in the Developing Anterior Chambers of Chick and Xenopus Embryonic Eyes. Developmental Biology, 68, 472-486. https://doi.org/10.1016/0012-1606(79)90219-7

[14] Halfter, W. and Schurer, B. (1994) A New Heparan Sulfate Proteoglycan in the Extracellular Matrix of the Developing Chick Embryo. Experimental Cell Research, 214, 285-296. https://doi.org/10.1006/excr.1994.1260

[15] Danysh, B.P. and Duncan, M.K. (2009) The Lens Capsule. Experimental Eye Research, 88, 151-164. https://doi.org/10.1016/j.exer.2008.08.002

[16] Hamburger, V. and Hamilton, H.L. (1951) A Series of Normal Stages in the Development of the Chick Embryo. Journal of Morphology, 88, 49-92. https://doi.org/10.1002/jmor.1050880104

[17] Martin, C., Alonso, M.I., Santiago, C., Moro, J.A., De la Mano, A., Carretero, R. and Gato, A. (2009) Early Embryonic Brain Development in rats Requires the Trophic Influence of Cerebrospinal Fluid. International Journal of Developmental Neuroscience, 27, 733-740. https://doi.org/10.1016/j.ijdevneu.2009.06.002

[18] Murdoch, A.D. and Iozzo, R.V. (1993) Perlecan: The Multidomain Heparan Sulphate Proteoglycan of Basement Membrane and Extracellular Matrix. Virchows Archiv A, 
423, 237-242. https://doi.org/10.1007/BF01606885

[19] Timpl, R. (1993) Proteoglycans of Basement Membranes. Experientia, 49, 417-428. https://doi.org/10.1007/BF01923586

[20] Timpl, R. (1994) Proteoglycans of Basement Membranes. EXS, 70, 123-144. https://doi.org/10.1007/978-3-0348-7545-5_8

[21] Lugemwa, F.N. and Esko, J.D. (1991) Estradiol Beta-D-Xyloside, an Efficient Primer for Heparan Sulfate Biosynthesis. Journal of Biological Chemistry, 266, 6674-6677.

[22] Govindraj, P., West, L., Smith, S. and Hassell, J.R. (2006) Modulation of FGF-2 Binding to Chondrocytes from the Developing Growth Plate by Perlecan. Matrix Biology, 25, 232-239. https://doi.org/10.1016/j.matbio.2006.01.003

[23] Schulz, M.W., Chamberlain, C.G., de Iongh, R.U. and McAvoy, J.W. (1993) Acidic and Basic FGF in Ocular Media and Lens: Implications for Lens Polarity and Growth Patterns. Development, 118, 117-126.

[24] Haloui, Z., Jeanny, J.C., Jonet, L., Courtois, Y. and Laurent, M. (1988) Immunochemical Analysis of Extracellular Matrix during Embryonic Lens Development of the Cat Fraser Mouse. Experimental Eye Research, 46, 463-474. https://doi.org/10.1016/S0014-4835(88)80004-6

[25] Lovicu, F.J. and McAvoy, J.W. (1993) Localization of Acidic Fibroblast GrowthFactor, Basic Fibroblast Growth-Factor, and Heparan-Sulfate Proteoglycan in Rat Lens-Implications for Lens Polarity and Growth-Patterns. Investigative Ophthalmology \& Visual Science, 34, 3355-3365.

[26] Soulintzi, N. and Zagris, N. (2007) Spatial and Temporal Expression of Perlecan in the Early Chick Embryo. Cells Tissues Organs, 186, 243-256.

https://doi.org/10.1159/000107948

[27] Moro-Balbas, J.A., Gato, A., Alonso, M.I., Martin, P. and de la Mano, A. (2000) Basal Lamina Heparan Sulphate Proteoglycan Is Involved in Otic Placode Invagination in Chick Embryos. Anatomy and Embryology, 202, 333-343. https://doi.org/10.1007/s004290000119

[28] Schwartz, N.B., Galligani, L., Ho, P.L. and Dorfman, A. (1974) Stimulation of Synthesis of Free Chondroitin Sulfate Chains by Beta-D-Xylosides in Cultured Cells. Proceedings of the National Academy of Sciences of the United States of America, 71, 4047-4051. https://doi.org/10.1073/pnas.71.10.4047

[29] Peterson, P.E., Pow, C.S., Wilson, D.B. and Hendrickx, A.G. (1995) Localisation of Glycoproteins and Glycosaminoglycans during Early Eye Development in the Macaque. Journal of Anatomy, 186, 31-42.

[30] Ring, C., Lemmon, V. and Halfter, W. (1995) Two Chondroitin Sulfate Proteoglycans Differentially Expressed in the Developing Chick Visual System. Developmental Biology, 168, 11-27. https://doi.org/10.1006/dbio.1995.1057

[31] Yao, R.H., Alcala, J. and Maisel, H. (1996) Developmental Changes in Glycoconjugate Composition during Chick Lens Morphogenesis. Experimental Eye Research, 62, 419-431. https://doi.org/10.1006/exer.1996.0047

[32] Van Rybroek, J.J. and Olson, M.D. (1981) Surface Coat Material Associated with the Cells of the Developing Lens Vesicle in the Chick Embryo. Anatomical Record, 201, 261-271. https://doi.org/10.1002/ar.1092010206

[33] Gato, A., Moro, J.A., Alonso, M.I., Pastor, J.F., Represa, J. and Barbosa, E. (1993) Chondroitin Sulfate Proteoglycan and Embryonic Brain Enlargement in the Chick. Anatomy and Embryology, 188, 101-106. https://doi.org/10.1007/BF00191455

[34] Knox, S., Merry, C., Stringer, S., Melrose, J. and Whitelock, J. (2002) Not All Perlecans Are Created Equa: Interactions with Fibroblast Growth Factor (FGF) 2 
and FGF Receptors. Journal of Biological Chemistry, 277, 14657-14665.

[35] Rossi, M., Morita, H., Sormunen, R., Airenne, S., Kreivi, M., Wang, L., Fukai, N., Olsen, B.R., Tryggvason, K. and Soininen, R. (2003) Heparan Sulfate Chains of Perlecan Are Indispensable in the Lens Capsule but Not in the Kidney. EMBO Journal, 22, 236-245. https://doi.org/10.1093/emboj/cdg019

[36] Tholozan, F.M., Gribbon, C., Li, Z., Goldberg, M.W., Prescott, A.R., McKie, N. and Quinlan, R.A. (2007) FGF-2 Release from the Lens Capsule by MMP-2 Maintains Lens Epithelial Cell Viability. Molecular Biology of the Cell, 18, 4222-4231. https://doi.org/10.1091/mbc.E06-05-0416

[37] Tholozan, F.M. and Quinlan, R.A. (2007) Lens Cells: More than Meets the Eye. International Journal of Biochemistry \& Cell Biology, 39, 1754-1759. https://doi.org/10.1016/j.biocel.2007.06.021

[38] McAvoy, J.W., Stump, R.J.W., Ang, S., Chen, Y. and Lovicu, F.J. (2005) Growth Factor Regulation of Lens Development: Key Roles for FGF and Wnt in Promoting Lens Cell Differentiation and Polarity. Mechanisms of Development, 122, S99-S99.

[39] Dong, S., Landfair, J., Balasubramani, M., Bier, M.E., Cole, G. and Halfter, W. (2002) Expression of Basal Lamina Protein mRNAs in the Early Embryonic Chick Eye. Journal of Comparative Neurology, 447, 261-273. https://doi.org/10.1002/cne.10245

[40] Le, A.C.N. and Musil, L.S. (2001) FGF Signaling in Chick Lens Development. Developmental Biology, 233, 394-411. https://doi.org/10.1006/dbio.2001.0194

[41] Lovicu, F.J. and McAvoy, J.W. (2005) Growth Factor Regulation of Lens Development. Developmental Biology, 280, 1-14. https://doi.org/10.1016/j.ydbio.2005.01.020

[42] Wride, M.A. (1996) Cellular and Molecular Features of Lens Differentiation: A Review of Recent Advances. Differentiation, 61, 77-93. https://doi.org/10.1046/j.1432-0436.1996.6120077.x

[43] Chamberlain, C.G. and McAvoy, J.W. (1997) Fibre Differentiation and Polarity in the Mammalian Lens: A Key Role for FGF. Progress in Retinal and Eye Research, 16, 443-478. https://doi.org/10.1016/S1350-9462(96)00034-1

[44] McAvoy, J.W., Chamberlain, C.G., Deiongh, R.U., Richardson, N.A. and Lovicu, F.J. (1991) The Role of Fibroblast Growth-Factor in Eye Lens Development. Annals of the New York Academy of Sciences, 638, 256-274. https://doi.org/10.1111/j.1749-6632.1991.tb49036.x

[45] Iyengar, L., Patkunanathan, B., McAvoy, J.W. and Lovicu, F.J. (2009) Growth Factors Involved in Aqueous Humour-Induced Lens Cell Proliferation. Growth Factors, 27, 50-62. https://doi.org/10.1080/08977190802610916

[46] Dawes, L.J., Sugiyama, Y., Lovicu, F.J., Harris, C.G., Shelley, E.J. and McAvoy, J.W. (2014) Interactions between Lens Epithelial and Fiber Cells Reveal an Intrinsic Self-Assembly Mechanism. Developmental Biology, 385, 291-303. https://doi.org/10.1016/j.ydbio.2013.10.030

[47] Mott, J.D. and Werb, Z. (2004) Regulation of Matrix Biologyogy by Matrix Metalloproteinases. Current Opinion in Cell Biology, 16, 558-564. https://doi.org/10.1016/j.ceb.2004.07.010

[48] Goodger, S.J., Robinson, C.J., Murphy, K.J., Gasiunas, N., Harmer, N.J., Blundell, T.L., Pye, D.A. and Gallagher, J.T. (2008) Evidence That Heparin Saccharides Promote FGF2 Mitogenesis through Two Distinct Mechanisms. Journal of Biological Chemistry, 283, 13001-13008. https://doi.org/10.1074/jbc.M704531200 
Submit or recommend next manuscript to SCIRP and we will provide best service for you:

Accepting pre-submission inquiries through Email, Facebook, LinkedIn, Twitter, etc. A wide selection of journals (inclusive of 9 subjects, more than 200 journals)

Providing 24-hour high-quality service

User-friendly online submission system

Fair and swift peer-review system

Efficient typesetting and proofreading procedure

Display of the result of downloads and visits, as well as the number of cited articles Maximum dissemination of your research work

Submit your manuscript at: http://papersubmission.scirp.org/

Or contact ojvm@scirp.org 